\title{
Half-metallicity, magnetic moments, and gap states in oxygen-deficient magnetite for spintronic applications
}

Cite as: Appl. Phys. Lett. 100, 032403 (2012); https://doi.org/10.1063/1.3678028

Submitted: 25 November 2011 . Accepted: 28 December 2011 . Published Online: 18 January 2012

R. Arras, L. Calmels, and B. Warot-Fonrose

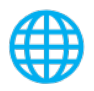

View Online

Export Citation

\section{ARTICLES YOU MAY BE INTERESTED IN}

Photoluminescence and photothermal effect of $\mathrm{Fe}_{3} \mathrm{O}_{4}$ nanoparticles for medical imaging and therapy

Applied Physics Letters 105, 091903 (2014); https://doi.org/10.1063/1.4895133

Influence of oxygen vacancies on the electronic structure and magnetic properties of $\mathrm{NiFe}_{2} \mathrm{O}_{4}$ thin films

Journal of Applied Physics 111, 093906 (2012); https://doi.org/10.1063/1.4704690

Preparation of highly conductive $\mathrm{Mn}$-doped $\mathrm{Fe}_{3} \mathrm{O}_{4}$ thin films with spin polarization at room temperature using a pulsed-laser deposition technique

Applied Physics Letters 86, 222504 (2005); https://doi.org/10.1063/1.1942640

Lake Shore CRYOTRONICS

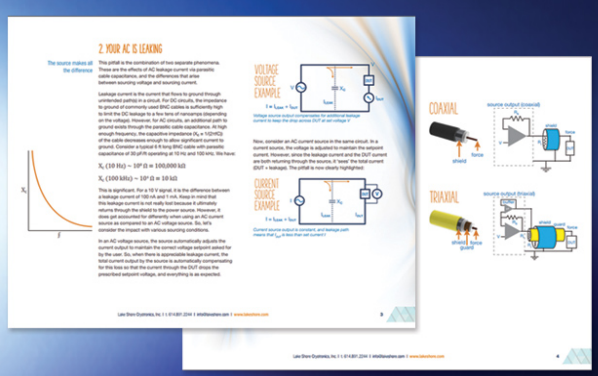

5 Electronic

- Measurement Pitfalls to Avoid

Get the whitepaper $\bullet$ 


\title{
Half-metallicity, magnetic moments, and gap states in oxygen-deficient magnetite for spintronic applications
}

\author{
R. Arras, ${ }^{\text {a) }}$ L. Calmels, and B. Warot-Fonrose \\ CEMES-CNRS, Université de Toulouse, 29 rue Jeanne Marvig, BP 94347, 31055 Toulouse Cedex 4, France
}

(Received 25 November 2011; accepted 28 December 2011; published online 18 January 2012)

\begin{abstract}
The electronic structure near oxygen vacancies in half-metallic magnetite has been calculated using first principles methods. Oxygen vacancies are responsible for the existence of gap states occupied by majority and minority spin electrons. We discuss whether these defects modify the spin magnetic moments, the magnetization, the magnetic coupling between $\mathrm{Fe}$ ions, and the half-metallic behaviour of magnetite. These results, which contribute to remove stumbling blocks to magnetite-based spintronic devices, could be useful to analyze the conductivity, the magnetotransport and magnetic properties, the electron and optical spectra of actual magnetite electrodes. (C) 2012 American Institute of Physics. [doi:10.1063/1.3678028]
\end{abstract}

Transition metal oxides form a class of materials with a huge variety of magnetic and electronic properties, which can be tuned when these materials are grown as thin layers. ${ }^{1,2}$ Half-metallic oxides, i.e., oxides with a metallic behaviour for one spin direction and an insulating behaviour for the other one, should in particular give access to huge magnetoresistance effects when used as magnetic electrodes in spintronic devices. Several half-metallic oxides have been considered for this reason, and a high spin-polarization has been measured in $\mathrm{La}_{1-\mathrm{x}} \mathrm{Sr}_{\mathrm{x}} \mathrm{MnO}_{3}\left(\right.$ Curie temperature $\left.\mathrm{T}_{\mathrm{C}}=370 \mathrm{~K}\right){ }^{3} \mathrm{Sr}_{2} \mathrm{FeMoO}_{6}$ $\left(\mathrm{T}_{\mathrm{C}}=410 \mathrm{~K}\right),{ }^{4,5} \mathrm{CrO}_{2}\left(\mathrm{~T}_{\mathrm{C}}=395 \mathrm{~K}\right),{ }^{6,7}$ and magnetite $\mathrm{Fe}_{3} \mathrm{O}_{4}$ between the Verwey temperature $(120 \mathrm{~K})$, and $\mathrm{T}_{\mathrm{C}}=858 \mathrm{~K}^{8}{ }^{8}$ The magnetoresistance measured in magnetite-based stacking is unfortunately far lower than the high value expected with half-metals. $^{9-11}$ All the possible explanations must be considered successively to identify the genuine reason for these bad performances and to break the deadlocks standing in the way of magnetite-based devices. Several studies focused on the modification of the physical properties of magnetite induced by structural defects like antiphase boundaries ${ }^{12-15}$ and cationic vacancies ${ }^{16,17}$ or by interfaces with an insulating barrier. ${ }^{18,19}$ We propose to study a new scenario to explain the low performances of magnetite-based devices, namely the modification of the electronic structure induced by oxygen vacancies in this oxide.

Changes induced by oxygen vacancies can be important in magnetic oxides, partly because these defects decrease the number of magnetic atom-O-magnetic atom bridges responsible for the superexchange and double exchange interactions which govern the magnetic order. First principles calculations have also confirmed that oxygen vacancies modify the magnetization of some magnetic oxides. ${ }^{20-22}$ The effects of oxygen vacancies have been studied experimentally in insulating spinel ferrites ${ }^{23-30}$ but not in half-metallic magnetite.

Bulk magnetite crystallizes in the inverse spinel structure above $120 \mathrm{~K}$ : its ionic description consists in $\mathrm{O}^{2-}$ ions arranged in a distorted face-centered-cubic ( $f c c$ ) lattice, which defines tetrahedral sites (A-sites) and octahedral sites

\footnotetext{
${ }^{\text {a) }}$ Author to whom correspondence should be addressed. Electronic mail: arras@cemes.fr.
}

(B-sites) in which $\mathrm{Fe}^{2+}$ and $\mathrm{Fe}^{3+}$ ions settle down according to the formula $\left[\mathrm{Fe}^{3+}\right]_{\mathrm{A}}\left[\mathrm{Fe}^{2+}, \mathrm{Fe}^{3+}\right]_{\mathrm{B}} \mathrm{O}_{4}^{2-}$. According to Hund laws, $\mathrm{Fe}^{2+}$ and $\mathrm{Fe}^{3+}$ ions, respectively, have a magnetic moment of 4 and $5 \mu_{\mathrm{B}}$ in this oxide. Fe ions in tetrahedral sites are antiferromagnetically coupled to those in octahedral sites, leading to a magnetic moment of $4 \mu_{\mathrm{B}}$ per formula unit. In the perfect crystal, any oxygen atom is bounded to $1 \mathrm{Fe}_{\mathrm{A}}$ and $3 \mathrm{Fe}_{\mathrm{B}}$ atoms. When the magnetite crystal contains an amount of oxygen vacancies corresponding to the formula $\mathrm{Fe}_{3} \mathrm{O}_{4-\delta}$, the oxidation degree of $\mathrm{Fe}$ atoms is reduced, two electrons being restored to the crystal for each neutral oxygen atom removed from the lattice. Consecutive changes in the magnetic moments of $\mathrm{Fe}_{\mathrm{A}}, \mathrm{Fe}_{\mathrm{B}}$, and $\mathrm{Fe}_{3} \mathrm{O}_{4}$ formula unit are not easy to guess. One could expect that each oxygen vacancy is responsible for the reduction, from $\mathrm{Fe}^{3+}$ to $\mathrm{Fe}^{2+}$, of two of the nearest neighbour $\mathrm{Fe}$ ions. However, the reduced $\mathrm{Fe}$ ions could be either the $\mathrm{Fe}_{\mathrm{A}}$ and one of the three first neighbour $\mathrm{Fe}_{\mathrm{B}}$ or two $\mathrm{Fe}_{\mathrm{B}}$ ions. In the first case, the restored electrons have opposite spin states and the magnetization of the crystal does not change. In the second case, the two electrons belong to minority spin bands, and the magnetic moment per formula unit decreases to $4(1-\delta / 2) \mu_{\mathrm{B}}$. In this discussion, we made the assumption that the global magnetic coupling between $\mathrm{Fe}$ atoms is qualitatively the same near an oxygen vacancy as in bulk magnetite. In the two possible situations described above, the restored electrons should occupy localized states with energy inside an energy gap of bulk magnetite (vacancy induced gap states). In any cases, the density of states (DOS) of magnetite may be strongly distorted by oxygen vacancies, the presence of which could even suppress the half-metallic behaviour. In this letter, we used first principles calculations to describe the modification of the electronic structure induced by diluted oxygen vacancies in magnetite. We show how these point defects modify the electron states, the magnetic moment of the metallic ions, and the magnetization of the crystal. We check whether vacancy induced gap-states exist and whether these states suppress the half-metallicity.

The supercell we used was built from the conventional cubic cell of magnetite containing 56 atoms or 8 formula units, in which we have removed 1 oxygen atom $(\delta=1 / 8)$. We neglected the fact that the lattice parameter may change 
with the vacancy content and we used the parameter of bulk magnetite $a=0.8397 \mathrm{~nm}$. We used the full potential linearized augmented plane wave code Wien2k (Ref. 31) and performed calculations within the local spin density approximation (LSDA), the LSDA $+U$ approximation, ${ }^{32}$ and with the hybrid functional PBE0 (Refs. 33 and 34) as implemented in Wien $2 \mathrm{k} .{ }^{35}$ These two last approximations correct the spurious self-interaction and have given similar results for bulk magnetite. ${ }^{15}$ For the LSDA $+U$ calculations, we used the parameters $U=4.5 \mathrm{eV}$ and $J=0.56 \mathrm{eV}$ proposed by Anisimov $^{36}$ and Novák. ${ }^{37}$ We used an energy cut-off of 25 Ry for the plane wave expansion of the Kohn-Sham wave functions in the interstitial area. The irreducible wedge of the first Brillouin zone was sampled with 60 Bloch vectors. The input data for the first iteration of each self-consistent calculation consist in the superposition of spin densities calculated for isolated atoms. These spin densities have been calculated using the spin populations which ensure an antiferromagnetic coupling between all the $\mathrm{Fe}_{\mathrm{A}}$ and $\mathrm{Fe}_{\mathrm{B}}$ atoms, except atoms close to a vacancy for which different initial spin populations have been tried. These different trials allowed rejecting metastable magnetic ordering and give access to the ground state which correctly describes the magnetic coupling between Fe atoms near the vacancy. The relaxation of the atomic positions was performed and stopped when the force applied on each atom was lower than $1.5 \mathrm{eV} / \AA$. We checked that our conclusions on the physical properties of oxygen deficient magnetite remain valid for vacancy contents up to $\delta=1 / 2$ (increasing $\delta$ only results in a widening of the gap-state peaks in the DOS curves). In the following, we only discuss in details the results obtained for $\delta=1 / 8$.

Our calculations have shown that the relaxation of the atom coordinates mainly concerns $\mathrm{Fe}_{\mathrm{A}}$ and $\mathrm{Fe}_{\mathrm{B}}$ ions in the vicinity of the vacancy: the bounding lengths are reduced by $1 \%$ between the $\mathrm{Fe}_{\mathrm{B}}$ atoms first neighbour of the vacancy and the oxygen atoms to which they are bounded and by $2.3 \%$ between the same oxygen atoms and $\mathrm{Fe}_{\mathrm{B}}$ atoms second neighbour of the vacancy. The closest $\mathrm{Fe}_{\mathrm{A}}$ ion is displaced along the [111] direction, by a distance of $0.22 \AA$ away from the vacancy. The modifications of the electronic structure induced by oxygen vacancies are described in Fig. 1, in which we compared the majority and minority spin total DOS of $\mathrm{Fe}_{3} \mathrm{O}_{3.875}$ (dark lines) and bulk $\mathrm{Fe}_{3} \mathrm{O}_{4}$ (red/light lines) calculated in the LSDA, LSDA $+U$, and PBE0 approximations. For better clarity, we have chosen to align in this figure the main band edges which appear in the total DOS curves of $\mathrm{Fe}_{3} \mathrm{O}_{3.875}$ and $\mathrm{Fe}_{3} \mathrm{O}_{4}$. Indeed, using the LSDA+U and PBE0 approximations, the position of the Fermi level $\mathrm{E}_{\mathrm{F}}$ with respect to band edges changes after removing an oxygen atom from the supercell. This shift of $E_{F}$ would have been negligible at a smaller oxygen vacancy content. From Fig. 1, we can very easily identify the defect-induced electron states which appear in the energy gaps of bulk magnetite; these occupied gap-states are responsible for the important shift of the Fermi level observed in the LSDA $+U$ and PBE0 calculations. The modification of the electronic structure induced by oxygen vacancies is more important for the PBE0 than for the LSDA $+U$ approximation. In particular, the shift of the Fermi level of $\mathrm{Fe}_{3} \mathrm{O}_{3.875}$ with respect to the unoccupied band edge of $\mathrm{Fe}_{3} \mathrm{O}_{4}$ is of $1.04 \mathrm{eV}$ for the

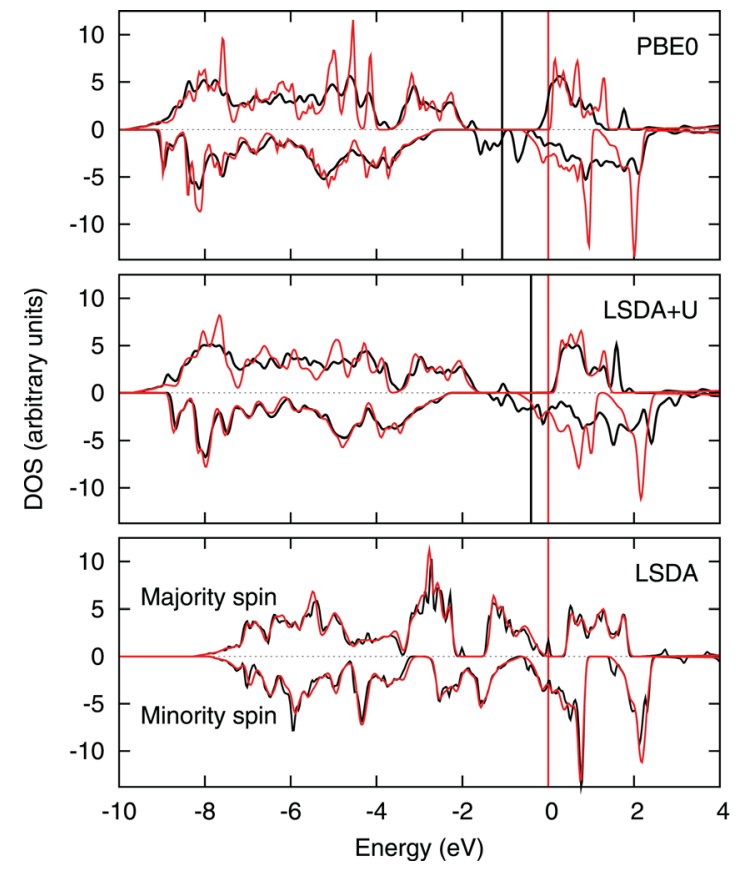

FIG. 1. (Color online) Total DOS of $\mathrm{Fe}_{3} \mathrm{O}_{3.875}$ (dark curves) and $\mathrm{Fe}_{3} \mathrm{O}_{4}$ (red/light curves). From the bottom to the top, the different panels correspond to the LSDA, LSDA $+U$, and PBE0 calculations. The DOS curves of $\mathrm{Fe}_{3} \mathrm{O}_{3.875}$ have been shifted to align the main band edges to those of bulk magnetite. The Fermi level of $\mathrm{Fe}_{3} \mathrm{O}_{3.875}$ and $\mathrm{Fe}_{3} \mathrm{O}_{4}$ are indicated by dark and red vertical lines.

PBE0 calculation and of $0.39 \mathrm{eV}$ with LSDA $+U$. These two approximations, however, give rather similar results for the energy of the occupied gap-states with respect to $\mathrm{E}_{\mathrm{F}}$ : for majority spin, these states appear at $0.7 \mathrm{eV}$ for PBE0 and at $0.65 \mathrm{eV}$ for $\mathrm{LSDA}+U$ below $\mathrm{E}_{\mathrm{F}}$. For minority spin, the energy range where occupied gap states can be found below $\mathrm{E}_{\mathrm{F}}$ has a width of $0.8 \mathrm{eV}$ for PBE0 and of $1.17 \mathrm{eV}$ for $\mathrm{LSDA}+U$. The PBE0 and LSDA $+U$ results show that the half-metallic character of magnetite is not destroyed at the relatively small content of oxygen vacancies considered here. The minority spin DOS at $\mathrm{E}_{\mathrm{F}}$ is however lower for $\mathrm{Fe}_{3} \mathrm{O}_{3.875}$ than for perfect bulk magnetite. It means that the magnetoresistive properties of magnetite-based devices should keep very high values, even if oxygen vacancies reduce the conductivity of the magnetite electrodes. The majority spin energy gap at $\mathrm{E}_{\mathrm{F}}$ is narrowed by occupied gapstates, from $1.98 \mathrm{eV}$ in $\mathrm{Fe}_{3} \mathrm{O}_{4}$ to $1.25 \mathrm{eV}$ in $\mathrm{Fe}_{3} \mathrm{O}_{3.875}$ in the PBE0 calculation, and from $1.91 \mathrm{eV}$ to $0.86 \mathrm{eV}$ with the LSDA $+U$ approximation. This narrowing of the energy gap should be observed in optical spectra. The advanced approximations PBE0 and LSDA $+U$ give similar DOS curves (with differences which could be reduced by adjusting the value of $U$ and $J$ ). This is not the case of the LSDA approximation, which does not correct the spurious self-interaction and does not efficiently describe the localization of Fe $d$-orbitals, thus giving narrower energy gaps even for bulk magnetite. The occupied gap-states induced by vacancies are only shown by few very small DOS peaks with this approximation; one of these peaks even destroys the half-metallic character of magnetite.

Partial-DOS curves allow understanding which atoms are involved in the defect-induced gap states. Fig. 2 describes the contribution to the total DOS of the $\mathrm{Fe}_{\mathrm{A}}$ and of 


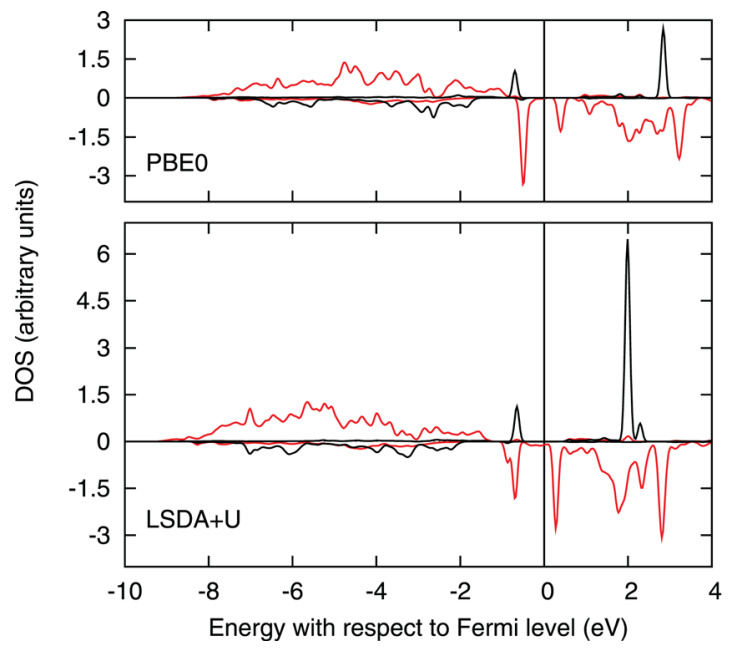

FIG. 2. (Color online) Contribution of the $\mathrm{Fe}_{\mathrm{A}}$ (dark curves) and of one the $3 \mathrm{Fe}_{\mathrm{B}}$ (red/light curves) first-neighbour of the vacancy to the total density of states. The bottom and the top panels, respectively, correspond to the $\mathrm{LSDA}+U$ and PBE0 calculations.

one of the three $\mathrm{Fe}_{\mathrm{B}}$ atoms the closest from the vacancy. The PBE0 and LSDA $+U$ results show that the occupied majority spin gap-states near $0.7 \mathrm{eV}$ below $\mathrm{E}_{\mathrm{F}}$ mainly involve the closest $\mathrm{Fe}_{\mathrm{A}}$ atom, while the minority spin gap-states at 0.5 $0.8 \mathrm{eV}$ below $\mathrm{E}_{\mathrm{F}}$ belong to the $\mathrm{Fe}_{\mathrm{B}}$ atoms first neighbour of a vacancy. This result confirms the ionic scenario in which the two electrons restored to the crystal per oxygen vacancy have different spin directions and are, respectively, captured by the closest $\mathrm{Fe}_{\mathrm{A}}{ }^{3+}$ and $\mathrm{Fe}_{\mathrm{B}}{ }^{3+}$ ions.

We found a similar ground state magnetic configuration for $\mathrm{Fe}_{3} \mathrm{O}_{3.875}$ than for $\mathrm{Fe}_{3} \mathrm{O}_{4}$ : the antiferromagnetic coupling between $\mathrm{Fe}_{\mathrm{A}}$ and $\mathrm{Fe}_{\mathrm{B}}$ atoms also holds in the vicinity of the vacancies, despite the suppression of some $\mathrm{Fe}-\mathrm{O}-\mathrm{Fe}$ bridges. The calculations also confirmed that the whole magnetization is the same for $\mathrm{Fe}_{3} \mathrm{O}_{3.875}$ and $\mathrm{Fe}_{3} \mathrm{O}_{4}$. With the PBE0 hybrid functional, the absolute value of the spin magnetic moments of the $\mathrm{Fe}_{\mathrm{A}}$ and $\mathrm{Fe}_{\mathrm{B}}$ ions are, respectively, $0.54 \mu_{\mathrm{B}}$ and $0.27 \mu_{\mathrm{B}}$ lower close to the vacancy than in bulk magnetite. This lowering is of $0.41 \mu_{\mathrm{B}}$ and $0.2 \mu_{\mathrm{B}}$ with the LSDA $+U$ approximation. Again, the modification of the spin magnetic moments and the constant magnetization support the hypothesis that a majority spin electron restored by the oxygen vacancy is captured by the closest $\mathrm{Fe}_{\mathrm{A}}{ }^{3+}$ ion, while a minority spin restored electron belongs to one of the closest $\mathrm{Fe}_{\mathrm{B}}{ }^{3+}$ ions.

In conclusion, we have shown that electrons restored by oxygen vacancies to the magnetite crystal occupy gap states in the main energy gaps below $\mathrm{E}_{\mathrm{F}}$. Electrons occupying majority and minority spin gap states are, respectively, captured by the closest $\mathrm{Fe}_{\mathrm{A}}$ and $\mathrm{Fe}_{\mathrm{B}}$ atoms. These vacancy states are responsible for a decrease of the spin magnetic moments of the closest $\mathrm{Fe}$ atoms and for a narrowing of the energy gap (which should be observed in optical or photoemission experiments). These structural defects, however, do not modify the magnetization of the crystal and the ferrimagnetic ordering. The half-metallic behaviour is preserved in oxygen deficient magnetite, even if the minority spin DOS is smaller at $\mathrm{E}_{\mathrm{F}}$. Consequently, the hypothetical presence of oxygen vacancies could explain the relatively small conductivity of magnetite but not the disappointing magnetoresistance performances of magnetite-based spintronic devices.
The calculations presented in this article have been performed at the CALMIP/UPS Toulouse parallel computer centre.

${ }^{1}$ M. Bibes and A. Barthelemy, IEEE Trans. Electron Devices 54, 1003 (2007). ${ }^{2}$ M. Bibes, J. E. Villegas, and A. Barthelemy, Adv. Phys. 60, 5 (2011).

${ }^{3}$ M. Bowen, M. Bibes, A. Bertelemy, J. P. Contour, A. Anane, Y. Lemaître, and A. Fert, Appl. Phys. Lett. 82, 233 (2003).

${ }^{4}$ K. Kobayashi, T. Kimura, H. Sawada, K. Terakura, and Y. Tokura, Nature 395, 6703 (1998).

${ }^{5}$ D. Serrate, J. M. de Teresa, and M. R. Ibarra, J. Phys. Condens. Matter. 19, 023201 (2007).

${ }^{6}$ W. J. De Sisto, P. R. Broussard, B. E. Nadgorny, and M. S. Osofsky, Appl. Phys. Lett. 76, 3789 (2000).

${ }^{7}$ A. Anguelouch, A. Gupta, G. Xiao, D. W. Abraham, Y. Ji, S. Ingvarsson, and C. L. Chien, Phys. Rev. B 64, 180408(R) (2000).

${ }^{8}$ Y. S. Dedkov, U. Rüdinger, and G. Güntherodt, Phys. Rev. B 65, 064417 (2002).

${ }^{9}$ X. W. Li, A. Gupta, G. Xiao, W. Qian, and V. P. Dravid, Appl. Phys. Lett. 73, 3282 (1998).

${ }^{10}$ P. J. van der Zaag, P. J. H. Bloemen, J. M. Gaines, R. M. Wolf, P. A. A. van der Heiden, R. J. M. van Veerdonk, and W. J. M. de Jonge, J. Magn. Magn. Matter. 211, 301 (2000).

${ }^{11}$ F. Greullet, E. Snoeck, C. Tiusan, M. Hehn, D. Lacour, O. Lenoble, C. Magen, and L. Calmels, Appl. Phys. Lett. 92, 053508 (2008).

${ }^{12}$ D. T. Margulies, F. T. Parker, M. L. Rudee, F. E. Spada, J. N. Chapman, P. R. Aitchison, and A. E. Berkowitz, Phys. Rev. Lett. 79, 5162 (1997).

${ }^{13}$ W. Eerenstein, T. T. M. Palstra, T. Hibma, and S. Celotto, Phys. Rev. B 66, 201101 (R) (2002).

${ }^{14}$ R. Arras, L. Calmels, and B. Warot-Fonrose, Phys. Rev. B 81, 104422 (2010).

${ }^{15}$ R. Arras, L. Calmels, and B. Warot-Fonrose, J. Phys.: Conf. Ser. 200, 032004 (2010).

${ }^{16}$ Z. Kakol and J. M. Honig, Phys. Rev. B 40, 9090 (1989).

${ }^{17}$ V. Chlan, P. Novák, H. Štěpánková, R. Řezníček, K. Kouřil, and A. Kozłowski, J. Magn. Magn. Mater. 322, 1079 (2010).

${ }^{18}$ R. Arras, L. Calmels, and B. Warot-Fonrose, IEEE Trans. Magn. 46, 1730 (2010).

${ }^{19}$ R. Arras, L. Calmels, and B. Warot-Fonrose, J. Phys.: Conf. Ser. 200, 072008 (2010).

${ }^{20}$ D. Stoeffler and S. Collis, J. Phys. Condens. Matter. 17, 6415 (2005).

${ }^{21}$ I. R. Shein, V. L. Kozhevnikov, and A. L. Ivanovskii, J. Phys. Chem. Solids 67, 1436 (2006).

${ }^{22}$ V. I. Anisimov, M. A. Korotin, I. A. Nekrasov, A. S. Mylnikova, A. V. Lukoyanov, J. L. Wang, and Z. Zeng, J. Phys. Condens. Matter. 18, 1695 (2006).

${ }^{23}$ U. Lüders, M. Bibes, J. F. Bobo, M. Cantoni, R. Bertacco, and J. Fontcuberta, Phys. Rev. B 71, 134419 (2005).

${ }^{24}$ A. Marcu, T. Yanagida, K. Nagashima, H. Tanaka, and T. Kawai, J. Appl. Phys. 102, 023713 (2007).

${ }^{25}$ Y. F. Chen, D. Spoddig, and M. Ziese, J. Phys. D: Appl. Phys. 41, 205004 (2008).

${ }^{26}$ X. Zuo, A. Yang, S. D. Yoon, J. A. Christodoulides, V. G. Harris, and C. Vittoria, Appl. Phys. Lett. 87, 152505 (2005).

${ }^{27}$ J. P. Zhou, H. C. He, and C. W. Nan, Appl. Surf. Sci. 253, 7456 (2007).

${ }^{28}$ R. Sayed Hassan, N. Viart, C. Ulhaq-Bouillet, J. L. Loison, G. Versini, J. P. Vola, O. Crégut, G. Pourroy, D. Muller, and D. Chateigner, Thin Solid Films 515, 2943 (2007).

${ }^{29}$ A. V. Ramos, T. S. Santos, G. X. Miao, M. J. Guittet, J. B. Moussy, and J. S. Moodera, Phys. Rev. B 78, 180402(R) (2008).

${ }^{30}$ A. Yang, Z. Chen, X. Zuo, D. Arena, J. Kirkland, C. Vittoria, and V. G. Harris, Appl. Phys. Lett. 86, 252510 (2005).

${ }^{31}$ P. Blaha, K. Schwarz, G. Madsen, D. Kvasnicka, and J. Luitz, Wien2k, An Augmented Plane Wave + Local Orbitals Program for Calculating Crystal Properties (Karlheinz Schwarz, Vienna, Austria, 2001).

${ }^{32}$ V. I. Anisimov, I. V. Solovyev, M. A. Korotin, M. T. Czyżk, and G. A. Sawatzky, Phys. Rev. B 48, 16929 (1993).

${ }^{33}$ M. Ernzerhof and G. E. Scuseria, J. Chem. Phys. 110, 5029 (1999).

${ }^{34}$ C. Adamo and V. Barone, J. Chem. Phys. 110, 6158 (1999).

${ }^{35}$ F. Tran, P. Blaha, K. Schwarz, and P. Novák, Phys. Rev. B 74, 155108 (2006).

${ }^{36}$ V. I. Anisimov, I. S. Elfimov, N. Hamada, and K. Terakura, Phys. Rev. B 54, 4387 (1996).

${ }^{37}$ P. Novák, F. Boucher, P. Gressier, P. Blaha, and K. Schwarz, Phys. Rev. B 63, 235114 (2001). 